\title{
Primary immunodeficiency diseases: developing news
}

\author{
Arnoldo Quezada, M.D. \\ Professor, Department of Pediatrics, Medicine School, University of Chile
}

The Expert Committee of the International Union of Immunological Societies updated periodically the classification of Primary Immunodeficiencies (PIDs) with the aim to increase knowledge, facilitate identification, and promote best or most favorable treatment for patients with these pathologic conditions. The last version divided PIDs in 9 major groups, including the Predominantly Antibody Deficiencies which are the most frequent cases in many reports. Genetic basis and molecular diagnosis have contributed to relevant aid in classification and diagnosis so that the majority of syndromes and entities are included in Online Mendelian Inheritance in Man (OMIM).

Specific antibody deficiency with normal immunoglobulin concentrations (SAD) and normal numbers of B cell is a PID with clinical manifestations similar to those of other predominant antibody deficiencies, often presenting as recurrent upper and lower respiratory tract infections due to capsulated bacteria, but some patients present with associated severe and life threatening infections that require admission and multiple antibiotic treatment. Reduced ability to produce antibodies to specific polysaccharide antigens is the hallmark of SAD, but their inheritance and definitive genetic defect involved in pathogenesis remain currently only in part explored. Diagnosis of SAD is established in children older than 2 years old by measurement of level of anti-capsular Immunoglobulin G specific for various serotypes of Streptococcus pneumoniae after immunization with polysaccharide pneumococcal vaccine. SAD was first reported in the early 1980s, and can be found in association with various primary and secondary immunodeficiency, in patients with dysmorphic syndromes or chromosomal abnormalities associated with recurrent infections, as well as other varied conditions, suggesting that there may be different abnormal pathways and different pathogenic mechanisms. Also, according to clinical severity and magnitude or persistence of immunological response to stimulation with polysaccharide antigens contained in pneumococcal vaccine, probably various forms or phenotype of SAD exists. However, in some patients especially those with more severe manifestations a strict correlate with the immune phenotype not always has been confirmed. Therefore, there may be a need for a better definition of the various phenotypes. Reduction in percentages of switched memory B cells and IgM memory B cells in patients with classic SAD compared with normal controls and children with deficient response to conjugated pneumococcal vaccine has been reported. On the other hand, switching memory B cells were considered a marker of poor prognosis associated with bronchiectasis and autoimmunity in patients with $\mathrm{SAD}$ or common variable deficiency.

Other issues still not widely agreed are post-vaccination IgG levels that must be considered appropriate, and the number of responses to serotypes that should be measured for the result to be considered reliable, as well as the percentage of positive responses depending on age group considered normal, although various practical applications in these fields have proved utility for predicting clinical course and selecting treatment options.

Clinical studies of SAD, especially in children, are scarce. Recurrent respiratory infections are the most common clinical manifestations reported but less frequent skin infections, systemic infections, chronic diarrhoea, and autoimmune disorder are described. Chronic otorrhoea, allergic rhinitis, asthma and any type of allergy have been also highlighted.

In other cases, many children with SAD over the age of three years responded adequately to revaccination with conjugate pneumococcal vaccine, so in some instances it may be concluded that the abnormality would be transient.

In many countries conjugate pneumococcal vaccine $(\mathrm{PCV})$ has been incorporated universally to national program of immunization and this situation would change the incidence of SAD, however in our hospital and teaching center as in other sites, children with inability to response to PCV have been identified. 
PIDs as specific syndromes are considered rare diseases while viewed as a group their frequency ranges around 1:2000 to 1:10000. Advances in the knowledge of molecular basis and pathophysiology of PIDs have achieved diagnosis and therapeutic strategies more precise. This new setting implies renewed challenges in the continual medical education and training of physicians caring potential patients with these diseases for increasing their awareness and to improve the management of PIDs.

\section{REFERENCES}

[1] Al-Herz W, Bousfiha A, Casanova JL, Chapel H, Conley ME, Cunningham-Rundles C, et al. Primary immunodeficiency diseases: an update on the classification from the International Union of Immunological Societies Expert Committee for Primary Immunodeficiency. Frontier Immunol. 2011; 2:1-26.

[2] Quezada A, Norambuena X, Inostroza J, Rodríguez J. Specific antibody deficiency with normal immunoglobulin concentration in children with recurrent respiratory infections. Allergol Immunopathol (Madr). 2015; 43(3):292-297. 